\title{
Regarding "Determining the Orientation of Directional Deep Brain Stimulation Electrodes Using 3D Rotational Fluoroscopy"
}

W e read with great interest the recently published article of Reinacher et $\mathrm{al}^{1}$ on the determination of the precise 3D orientation of directional leads for deep brain stimulation (DBS) with rotational fluoroscopy. We agree with the authors that for targeted directional steering of DBS the "detailed knowledge of the exact orientation of the electrode array with respect to its functional environment" is essential. We also like the idea of using not only the directional marker but also the signal of the segmented electrode contacts to improve the accuracy of the measured orientation angle. However, we would like to raise concerns about the solution presented and the conclusions drawn.

To describe the position of a directional lead in its functional environment requires at least 6 parameters, for example, the $\mathrm{X}, \mathrm{Y}$, $\mathrm{Z}$ coordinates of the lead tip and the 3 angles of lead orientation defined in 3D stereotactic space. Coordinates and angles of leads as they appear in a volumetric image may be transformed into another coordinate system by applying a transformation matrix comprising 3 translations along the coordinate axes and 3 rotations with respect to the coordinate axes. Furthermore, the location and shape of implants, as they appear in a sectional image, may be calculated from the intersection of the $3 \mathrm{D}$ object describing the implant itself and a plane representing the image geometry. For leads that do not intersect the plane at right angles, both shape and angles commonly incline; thus, the angles between the lines connecting the gaps among the 3 electrode segments are no longer $60^{\circ}$ but vary in the range of $40^{\circ}-80^{\circ} .^{2}$

The method described by the authors determines the directional angle of the lead as it projects into a plane defined by the path of the x-ray focus of the 3D-flouroscopy device used. In order to account for patient orientation during 3D fluoroscopy, measured angles were referenced to the patient's sagittal direction

http://dx.doi.org/10.3174/ajnr.A5377 as it appeared in the plane of the $\mathrm{x}$-ray focus path. The so-determined angle was then taken as the searched lead orientation angle, disregarding any inclinations due to oblique orientations of the lead inside the brain and the further tilt angles of the patient's head with respect to the imaging system.

Problems associated with the oblique and tilted courses of the leads were not addressed by the authors and not investigated in their experiments. Therefore, and also because the authors did not compare their results against a ground truth, we believe that the proposed method is insufficient for detecting "the exact orientation of the electrode array with respect to its functional environment."

Disclosures: Veerle Visser-Vandewalle-UNRELATED: Consultancy: Medtronic, Abbott, Boston Scientific; Payment for Lectures Including Service on speakers Bureaus: Medtronic, Abbott, Boston Scientific.

\section{REFERENCES}

1. Reinacher PC, Krüger MT, Coenen VA, et al. Determining the orientation of directional deep brain stimulation electrodes using 3D rotational fluoroscopy. AJNR Am J Neuroradiol 2017;38:1111-16 CrossRef Medline

2. Sitz A, Hoevels M, Hellerbach A, et al. Determining the orientation angle of directional leads for deep brain stimulation using computed tomography and digital x-ray imaging: a phantom study. Med Phys 2017 Jun 22. [Epub ahead of print] CrossRef Medline

(D). Treuer

(D) A. Hellerbach Department of Stereotactic and Functional Neurosurgery University Hospital of Cologne Cologne, Germany

(D). Borggrefe

Division of Neuroradiology, Institute of Diagnostic and Interventional Radiology University Hospital of Cologne Cologne, Germany (D) V. Visser-Vandewalle Department of Stereotactic and Functional Neurosurgery University Hospital of Cologne Cologne, Germany 